\title{
Corroboration of Dynamic Black Hole Gravitational Physics from Observations of Cygnus X-1
}

\section{The Gravitation of Spinning Black Holes}

\author{
Andrew Worsley ${ }^{1}$ \\ ${ }^{1}$ Fellow of the Royal Astronomical Society, Burlington House, Piccadilly, London W1J 0BQ, UK \\ Correspondence: Andrew Worsley, Fellow of the Royal Astronomical Society, Burlington House, Piccadilly, \\ London W1J 0BQ, UK. E-mail: andrew.worsely@kcl.ac.uk
}

Received: February 21, 2012

doi:10.5539/apr.v4n2p159

\author{
Accepted: March 6, 2012 Online Published: May 1, 2012 \\ URL: http://dx.doi.org/10.5539/apr.v4n2p159
}

\begin{abstract}
The compact black hole primary in the X-ray binary Cygnus X-1, has recently been characterised by observation. This data can be analysed to test models of black hole gravitational physics. Using the optimum observational data with the least fit parameters, the average of 3 spectral data sets revealed an averaged inner rim accretion disc radius $R_{i n}=2.24 \mathrm{GM} / \mathrm{c}^{2}$. Using the Kerr spinning black hole model results in a theoretical apparent reduction in $R_{i n}=1.41 \mathrm{GM} / \mathrm{c}^{2}$, which also results in an extremely relativistic spin parameter $a_{*}>0.97 \mathrm{c}$. Here the data is analysed using a dynamic gravitational model. Utilizing this model $R_{i n}=2.24 \mathrm{GM} / \mathrm{c}^{2}$, which is equivalent to the observed $R_{i n}=2.24 \mathrm{GM} / \mathrm{c}^{2}$. In this case the spin parameter does not reach such extreme relativistic speeds, at $a_{*}=0.76 \mathrm{c}$. These results using dynamic black hole physics are in very good agreement with direct observations of Cygnus X-1. Importantly, this model offers further testable predictions of black hole gravitational physics.
\end{abstract}

Keywords: spinning black hole, Schwarzschild radius, spin plot, gravitational physics, gravitational time dilation, Cygnus X-1

\section{Introduction}

\section{Spinning Black Holes}

Newly published data on the compact primary in Cygnus X-1, allows an examination of observational models of spinning black holes (Gou et al., 2011). The principal difference between the static Schwarzschild black hole and the spinning black hole, relates to the Schwarzschild radius and the event horizon. The standard solution to the equations of the general theory of relativity (GTR), which describe the event horizon of a non spinning Schwarzschild black hole (Straumann, 2004), is given in Eq. (1).

The Schwarzschild radius $\left(R_{S}\right)$

$$
R_{S}=2 G M / c^{2}
$$

where $G$ is the gravitational constant, $M$ is the mass and $c$ is the speed of light

In the non spinning Schwarzschild black hole described by the general theory of relativity, the event horizon, the escape velocity of light, and the Schwarzschild radius are by definition synchronous. However, more recently it has been surmised, both theoretically and experimentally, that most if not all black holes are spinning.

This has led to a reanalysis of the event horizon of the spinning black hole. In the standard Kerr spinning black hole model, there is an apparent theoretical reduction in the event horizon at the equator of the black hole to $1 \mathrm{GM} / \mathrm{c} 2$ (Kerr, 1963) In the standard spinning Kerr model this results in a theoretical reduction in the inner radius of the accretion disc. So that using the Kerr model, the measured Rin $=2.24 \mathrm{GM} / \mathrm{c} 2$, theoretically reduces to $\operatorname{Rin}=1.41 \mathrm{GM} / \mathrm{c} 2$ (Gou et al., 2011). Additionally, in the Kerr black hole model there is also a spin plot relating the inner radius (Rin), to the speed of the spinning black hole, and this spin becomes exceedingly relativistic (Bardeen, Press \& Tuelkolsky, 1972). These modelling issues have prompted a reanalysis of gravitational models and the physics of black holes.

In the previously published dynamic black hole gravitation presented here, the event horizon, the escape velocity of light and the Schwarzschild radius, remain as with the standard Schwarzschild black hole - synchronous even 
at the equator (Worsley, 2008). Equally in this model Rin is identified with the direct observations of radius of the innermost stable circular orbit RISCO. Thus in this gravitational model Rin $=2.24 \mathrm{GM} / \mathrm{c} 2$, which is exactly equivalent to the measured Rin $=2.24 \mathrm{GM} / \mathrm{c} 2$, as seen in Cygnus X-1 (Guo et al., .2011). Thus the newly published data on Cygnus X-1, corroborate the dynamic form of dynamic gravitational black hole physics presented here. Moreover, the dynamic element in this dynamic form of gravitation is essential to obtain the degree of accuracy needed with observations of spinning black holes.

These equations give a dynamic increase in gravity in the proximity of a black hole and at the Schwarzschild radius event horizon. Additionally, with the dynamic black hole gravitation both the event horizon and the Schwarzschild radius remain mathematically synchronous at $2 \mathrm{GM} / \mathrm{c} 2$, even in the spinning black hole. This dynamic gravitation thus has a number of advantages; this includes extreme accuracy, and the avoidance of the mathematical formation of infinitely dense singularities. Another advantage is that this dynamic increase enables the formulation of a spin plot, which does not yield extremely relativistic spin parameters for black holes.

Overall this dynamic gravitational physics can thus better account for the observed black hole Rin, for the compact primary black hole in Cygnus X-1. Importantly it also allows further testable predictions of black hole gravitational physics.

\section{Methods}

\section{Mathematical Methods}

The published data from studies on the spin of Cygnus X-1 are used to test black hole gravitation models (Gou et al., 2011). All mathematical calculations follow strict standard algebraic and standard mathematical rules. The gravitational equations used [Eqs. (1-3)], are those of the previously published adaptation to gravity as described in the introduction (Worsley, 2008). The dynamic increases in the force of gravity are given by the equations.

Acceleration due to dynamic gravity at the Schwarschild radius $\left(\mathrm{a}_{\mathrm{g}}\right)$

$$
\begin{gathered}
R_{S}=2 G M / c^{2} \\
a_{g}=\frac{G M}{R^{2}}\left[1+3 G M / R c^{2}\right]^{2} \\
a_{g}=\frac{G M}{R^{2}}[1+3 / 2]^{2}=a_{g}=\frac{G M}{R^{2}} x[6.25]
\end{gathered}
$$

where $\mathrm{M}$ is the larger mass, $\mathrm{c}$ is the speed of light and $\mathrm{G}$ the gravitational constant, $\mathrm{R}$ is the distance, (normally taken as the radius).

The principle physics proofs are based upon standard physical formulae. The gravitational equations used, offer a high degree of agreement with currently known values compared to experimental observation. The same equation also applies to data on gravitational time dilation as seen in global positioning systems (GPS). It has been shown that this dynamic gravity technically gives exactly the same results as gravitational observations, in the range where these have been widely tested (see Appendix A \& B, worked examples). This applies to the advance in the perihelion of Mercury and other planets in the solar system (Appendix A). The same equation also applies to data on gravitational time dilation as seen in global positioning systems (GPS), and is accurate to better than 1 part in $10^{11}$ (Appendix B).Very accurate results are also obtained with binary pulsars using this advanced dynamic gravitation (Worsley, 2008).

These technically give exactly the same answers as experimental observation, in low and medium mass density objects, where gravity has been widely tested. But in high mass density objects, such as black holes, the equations do not break down by resulting in the formation of singularities. The paper also proposes observational experiments for the experimental verification of the findings using observations of black holes, as listed in the results and conclusions (see also Table 1). They also allow the formulation of a spin plot around black holes based on the fraction of the speed of light, which can be tested (see Figure 1).

\section{Results}

\section{Calculating the Spin Parameter of Spinning Black Holes}

The results of the Very Long Baseline Array (VLBA) have enabled the characterization of the parameters of the compact primary in the binary Cygnus X-1. Because the data are only useful when the spectral hardness falls 
below a certain cut point $(\mathrm{SH}<0.7)$, these data can only be infrequently measured. These observations were first made in a 1996 spectral analysis SP1 (Cui et al., 1998), and again more recently twice in 2010, SP2 and SP3 (Guo et al., 2011). Using these data the calculated distance of Cygnus X-1, is $\mathrm{D}=1.86 \pm 0.12 \mathrm{kpc}$, the mass of the black hole has been estimated at $M=14.8 \pm 1.0$ solar masses, and the inclination $\mathrm{i}=27.1 \pm 0.8$ degrees. The optimal non Kerr model (model C) has the advantage of harmonizing the fitted values of the parameters of the thermal component $T_{i n}$. The values for the thermal component for SP1-3 were $T_{i n}=0.517,0.537$ and $0.539 \mathrm{keV}$ respectively. These data allow the calculation of the $R_{i n}$ and in turn the spin parameter $\mathrm{a} *$. The best model for the calculation of $R_{i n}$ (Model C) revealed an observed value of $2.30 \mathrm{GM} / \mathrm{c}^{2}$ for spectral analysis of the data set carried out in 1996 (SP1). More recently the two further spectral analyses carried out in 2010, SP2 and SP3 gave values of 2.32 and $2.10 \mathrm{GM} / \mathrm{c}^{2}$ respectively (Gou et al., 2011). The total averaged value for SP1-3, $R_{\text {in }}=2.24 \pm$ $0.122 \mathrm{GM} / \mathrm{c}^{2}$. Using this data, the Kerr spinning black hole model, results in an apparent reduction in $R_{i n}=1.41$ $\mathrm{GM} / \mathrm{c}^{2}$ and an extreme spin parameter $\mathrm{a} *>0.97 \mathrm{c}$ (Gou et al., 2011).

Here we reanalyze the data using a form of dynamic black hole gravitation, in accordance with Eq. 2. First it is possible to calculate the dynamic increase in the acceleration due to gravitation in the proximity of a spinning black hole, the tabulation of this is seen in Table 1. As the standard Schwarzschild radius $R_{S}=2 G M / c^{2}$ is being used in this model, the results are tabulated in multiples of $1 R_{S}$, out to a distance of $12 R_{S}$ (see Table 1). The results show a dynamic increase in the acceleration due to gravity in the proximity of a black hole, as the Schwarzschild radius is approached. The increase in the acceleration due to gravity results in an increase in angular velocity, giving an accretion disc spin plot as a fraction of the speed of light (see Figure 1).

Table 1 . Acceleration due to gravity (ag) and spin parameter $\left(a^{*}\right)$, in the proximity of a black hole, using dynamic Newtonian advanced gravitation (DNAg).

$$
\begin{aligned}
& R_{\text {in }}=12 R_{S}: \quad a_{g}=\frac{G M}{R^{2}} x[1.27], \quad a^{*}=0.041 c \\
& R_{\text {in }}=6 R_{S}: \quad a_{g}=\frac{G M}{R^{2}} \times[1.56], a^{*}=0.063 c \\
& R_{\text {in }}=3 R_{S}: \quad a_{g}=\frac{G M}{R^{2}} x[2.25], a^{*}=0.129 c \\
& R_{i n}=2.5 R_{S}: \quad a_{g}=\frac{G M}{R^{2}} x[2.56], \quad a^{*}=0.168 \\
& R_{\text {in }}=2 R_{S}: \quad a_{g}=\frac{G M}{R^{2}} x[3.06], a^{*}=0.240 \mathrm{c} \\
& R_{\text {in }}=1.75 R_{S}: \quad a_{g}=\frac{G M}{R^{2}} x[3.44], a^{*}=0.303 c \\
& R_{\text {in }}=1.5 R_{S}: \quad a_{g}=\frac{G M}{R^{2}} x[4.00], a^{*}=0.409 c \\
& R_{\text {in }}=1.25 R_{S}: \quad a_{g}=\frac{G M}{R^{2}} x[4.84], a^{*}=0.599 c \\
& R_{i n}=1.125 R_{S}: \quad a_{g}=\frac{G M}{R^{2}} x[5.44], a^{*}=0.758 c \\
& R_{\text {in }}=1.0625 R_{S}: \quad a_{g}=\frac{G M}{R^{2}} x[5.82], a^{*}=0.866 c \\
& R_{\text {in }}=1 R_{S}: \quad a_{g}=\frac{G M}{R^{2}} x[6.25], a^{*}=1.000 \mathrm{c}
\end{aligned}
$$

Where $R_{i n}$ is the inner radius of the accretion disc, $R_{S}$ is the standard Schwarzschild radius $R s=2 \mathrm{GM} / \mathrm{c}^{2}$. G is the gravitational constant, $M$ is the mass of the black hole, $c$ is the speed of light. Answers given to 3 decimal places. 


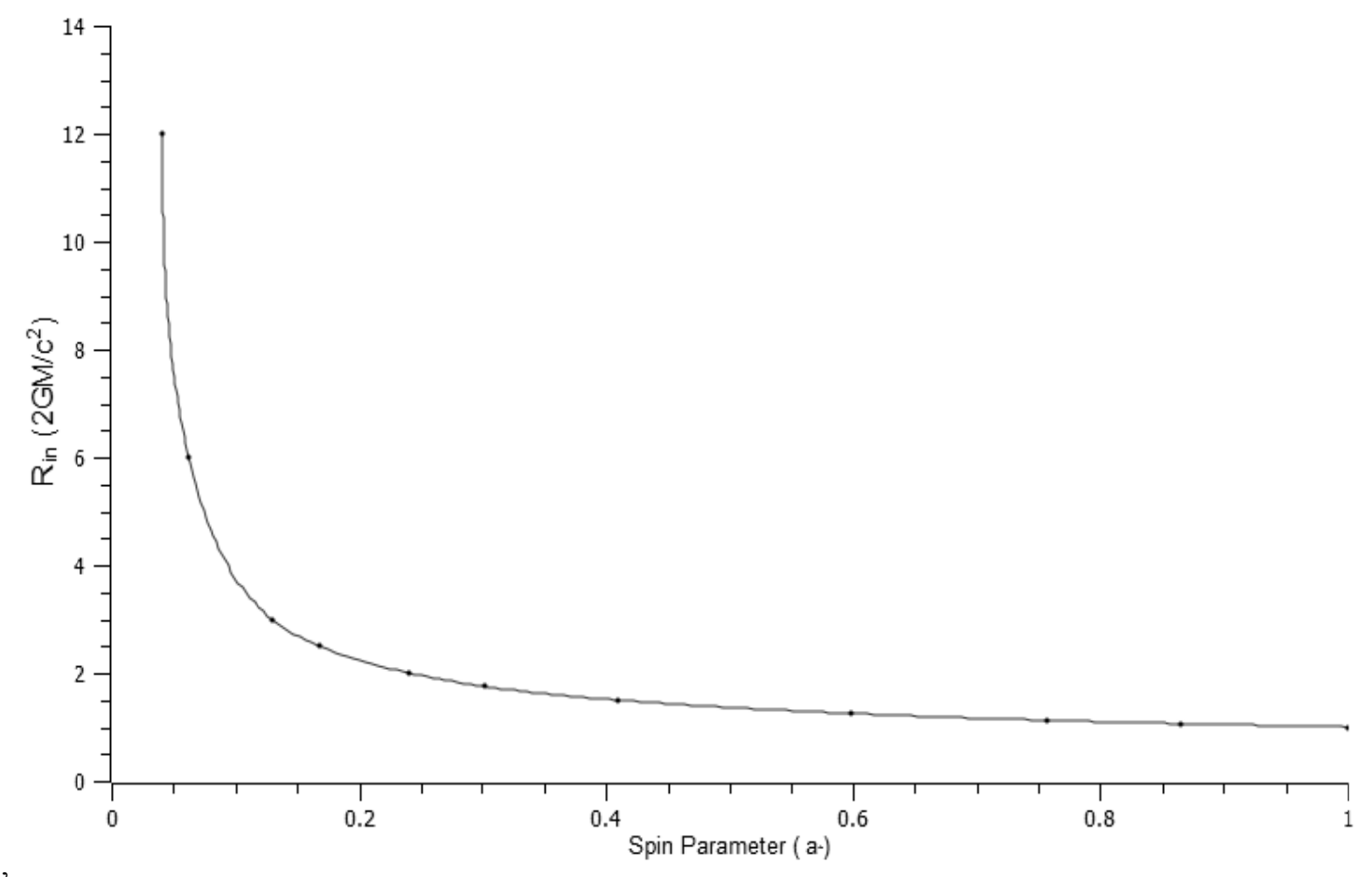

Figure 1. Shows the spin parameter $\mathrm{a} *$, in multiples of the speed of light vs. the accretion disc inner radius $R_{i n}$, in multiples of the standard Schwarzschild radius $R_{S}=2 \mathrm{GM} / \mathrm{c}^{2}$.

From here it is possible to take the observed estimate for $R_{\text {in }}=2.24 \mathrm{GM} / \mathrm{c}^{2}$, without further remodelling to allow for the Kerr spinning black hole model. So the Kerr model reduces the inner radius $R_{i n}=1.41 \mathrm{GM} / \mathrm{c}^{2}$, and thus arrives at an extreme spin parameter $\mathrm{a}_{*}>0.97 \mathrm{c}$. In the gravitational model used here, the observed $R_{\text {in }} 2.24$ $G M / c^{2}$ is used (see Table 1); this gives a spin parameter $\mathrm{a}_{*}=0.766 c$. For the spin parameter, the results are plotted in multiples of $1 R_{S}$, out to a distance of $12 R_{S}$ (see Figure 1).

In summary, using the spin plot in Figure 1, for Cygnus X-1 the observed $R_{i n}=2.24 G M / c^{2}$ is in exact agreement with the gravitational physics presented here. This equates directly to a spin parameter $\mathrm{a}_{*}=0.766 c$. Thus these results, using this black hole gravitation, are in very good agreement with direct observations of Cygnus X-1.

\section{Conclusions and Discussion}

\section{Corroboration of Advanced Black Hole Gravitational Physics}

A reanalysis of recently published data on Cygnus X-1, allows gravitational models of black hole physics to be tested. In this paper a novel form of dynamic black hole gravitation gives the best fit to the observed data (Worsley, 2008). This form of gravitational physics produces a dynamic increase in gravitation in the vicinity of a black hole. The dynamic element is essential to obtain the degree of accuracy obtained with spinning black holes. Indeed, it has already been shown that this dynamic form of gravity technically gives exactly the same results as GTR, in the range where GTR has been widely tested (see Appendices A \& B). The same exact agreement also applies, to a reanalysis of binary pulsar orbital data (Worsley, 2009). Where there is a clear advantage with the dynamic form of gravitation, is in the description of black hole physics.

When describing a black hole a large degree of success has been attributed to GTR. In black holes GTR predicts the standard Schwarzschild black hole radius $R_{S}=2 G M / c^{2}$. This is the boundary at which light is unable to escape and acts as the event horizon. However, in classical gravitation the actual black hole object itself then becomes an infinitely dense singularity. This presents some mathematical and conceptual problems. With the dynamic black hole gravitation presented here, the Schwarzschild radius is maintained but the infinitely dense singularity is obviated. This applies to small and super-massive black holes alike. Indeed, this dynamic gravitation has already allowed the accurate modelling of supermassive black holes and the presence of dark matter and gravitation lensing at the centre of galaxies and at the cosmological level (Cline, 2003; Worsley, 2011). Indeed these results technically agree exactly with the most recent estimates of cosmological dark matter (Kowalski, 
2008). Moreover this dynamic gravitational model is capable of explaining observations of the bullet cluster (Pointecouteau \& Silk, 2005; Worsley, 2012). Moreover, this gravitational physics has the advantage of being converted into quantum gravity (Worsley, 2009), in such a way as to also dovetail in with quantum electrodynamics (Worsley, 2010).

The next test for gravitational physics arises in the case of the spinning black hole, such as in Cygnus X-1. In the Kerr spinning black hole model, there is a theoretical divergence of the Schwarzschild radius and the event horizon (Kerr, 1963). Moreover, in the spin plot in the Kerr model, the Schwarzschild radius becomes asynchronous and rises to $R_{S}=6 G M / c^{2}$, at the "equator" of the black hole. Concurrently the event horizon is postulated to become half the $R_{S}$, seen in classical general relativity at the equator, so that $R_{S}=1 \mathrm{GM} / \mathrm{c}^{2}$. In the Kerr spinning black hole model, this means that the measured value for accretion disc inner radius $R_{i n}=2.24$ $G M / c^{2}$, needs to be further remodelled. This further remodelling to allow for the Kerr black hole, reduces the inner radius $R_{i n}=1.41 \mathrm{GM} / \mathrm{c}^{2}$. This in turn results in a highly relativistic extreme spin parameter at $0.97 \mathrm{c}$ (Guo et al., 2011).

With the dynamic model presented here the standard Schwarzschild radius is logically maintained as the event horizon $R_{S}=2 G M / c^{2}$. Equally the actual observed value for $R_{i n}=2.24 G M / c^{2}$, is in exact agreement with the experimental findings. Thus the spin parameter can then be calculated from the direct observations of $R_{i n}$ (see Table 1). This gives a far less extreme spin parameter of $0.76 c$, than does the Kerr model at $0.97 \mathrm{c}$. For comparison, the fastest known neutron star spin is $716 \mathrm{~Hz}$, which implies a spin parameter of just $0.1 \mathrm{c}$. It is unlikely therefore that such highly relativistic spin parameters exist in black holes. Moreover recent evidence suggests that the galactic halo may be predominantly composed of (primordial) spinning black holes (Hawkins, 2011). The presence of these black holes may also be better explained by the dynamic gravity presented here. More importantly, the gravitational model presented here also allows further directly testable observations of black holes using new and established methods (Schodel, 2002). This particularly applies to tests of supermassive black holes using a previously described model, and tests of binary black hole systems in the galactic halo (Worsley, 2012).

\section{References}

Bardeen, J. M., Press, W. H., \& Tuelkolsky, S. A. (1972). Rotating Black Holes: Locally Non rotating Frames, Energy Extraction and Scalar Synchrotron Radiation. Astrophys. J, 178, 347-369. http://dx.doi.org/10.1086/151796

Cline, D. (2003). $5^{\text {th }}$ International Symposium on the Detection of Dark matter and Dark Energy in the Universe. Nuc. Phys. B, Proceedings Supplement. edited by D. Cline. Elsevier Amsterdam.

Cui, W., et al. (1998). Simultaneous ASCA and RXTE Observations of Cygnus X-1During its 1996 State Transition. Astrophys J., 493, L75-L78. http://dx.doi.org/10.1086/311134

Gou, L., et al. (2011). The extreme Spin of the Black Hole in Cygnus X-1. arXiv:1106.3690.

Hawkins, M. R. S. (2011). The case for primordial black holes as dark matter, Mon. Not. Roy. Astron. Soc., 415, 2744. http://dx.doi.org/10.1111/j.1365-2966.2011.18890.x

Kerr, R. P. (1963). Gravitational field of a spinning mass as an example of algebraically special metrics. Phys. Rev. Lett., 11, 237. http://dx.doi.org/10.1103/PhysRevLett.11.237

Kowalski, M., et al. (2008). Improved Cosmological Constraints from New, Old and Combined Supernova Data Sets. Astrophys. J., 686, 749. http://dx.doi.org/10.1086/589937

Ohanian, H. C., \& Ruffini, R. (1994). Gravitation and Spacetime ( $2^{\text {nd }}$ Ed.). New York.: W. W Norton \& Co.

Pointecouteau, E., \& Silk, J. (2005). New Constraints on MOND from Galaxy Clusters. 364, 654-658

Schodel, R., et al. (2002). A star in a 15.2-year orbit about the super-massive black hole at the centre of the Milky Way. Nature, 494, 694. http://dx.doi.org/10.1038/nature01121

Straumann, N. (2004). General Relativity with Applications to Astrophysics. Springer Verlag, Berlin.

Worsley, A. (2008). An advanced dynamic adaptation of Newtonian equations of gravity. Phys. Essays, 21, 222-228. http://dx.doi.org/10.4006/1.3027501

Worsley, A. (2009). String quintessence and the formulation of advanced quantum gravity. Phys. Essays, 22, 364-377. http://dx.doi.org/10.4006/1.3182733

Worsley, A. (2010). Harmonic quintessence and the formulation of a fundamental energy equivalence equation. Phys. Essays, 23, 311-319. http://dx.doi.org/10.4006/1.3392799 
Worsley, A. (2011). Advances in Black Hole Gravitational Physics and Cold Dark Matter Modelling. Open Astron. J., 4, 6-13. Retrieved from http://www.benthamscience.com/open/toaaj/articles/V004/6TOAAJ.pdf

Worsley, A. (2012). Advances in Black Hole Physics and Dark Matter Modelling of the Galactic Halo. App. Phys. Res., 4(1), 128-137. http://dx.doi.org/10.5539/apr.v4n1p128

\section{Appendix A.}

\section{Advances in the Perihelia of Solar System Bodies}

1). Advance in the Perihelion of Mercury (Worked Example)

$$
\Delta \operatorname{circ}=3 G M S /\left(c^{2} a\left(1-e^{2}\right)\right)=7.987 \times 10^{-8}
$$

multiplied by the No. of orbits in a century,

$$
=\quad 3.316 \times 10^{-5}
$$

the ratio of circumference to arc second

$$
=360 \times 3600=1.296 \times 10^{6}
$$

calculated advance in the perihelion of Mercury per century

$$
3.316 \times 10^{-5} \times 1.296 \times 10^{6}=\underline{42.98 \mathrm{arc} \mathrm{sec}} .
$$

Equivalent general relativistic value per century

$$
=42.98 \text { arc sec }
$$

Experimentally estimated advance in the perihelion of Mercury per century (Ohanian \& Ruffini, 1994)

$$
=43 \pm 0.1 \text { arc sec }
$$

where $\Delta_{\text {circ }}$ is the change in circumference of the orbit of Mercury, G is the gravitational constant, Ms the mass of the Sun, $\mathrm{c}$ the speed of light, a is the semi major axis of Mercury 's orbit (in meters), e is the eccentricity.

2). Advance in the Perihelion of Mars (Worked Example)

change in the circumference of Mars orbit:

$$
\Delta \operatorname{circ}=3 G M S /\left(c^{2} a\left(1-e^{2}\right)\right)=1.9595 \times 10^{-8}
$$

multiplied by the number of Mars orbits in a century (53.15):

$$
=1.0416 \times 10^{-6}
$$

the ratio of circumference to arc second

$$
360^{\circ} \times 3600=1.296 \times 10^{6}
$$

calculated advance in the perihelion of Mars per century

$$
1.0416 \times 10^{-6} \times 1.296 \times 10^{6}=1.35 \mathrm{arcsec} / \mathrm{cy}
$$

Equivalent general relativistic value per century

$$
=1.35 \mathrm{arcsec} / \mathrm{cy}
$$

Experimentally estimated advance in the perihelion of Mars per century (Ohanian \& Ruffini, 1994).

$$
=1.35 \pm 0.1 \mathrm{arcsec} / \mathrm{cy}
$$

where $\Delta_{\text {circ }}$ is the change in circumference of the orbit of Mars, $\mathrm{G}$ is the gravitational constant, $M_{S}$ is the mass of the Sun, $\mathrm{c}$ the speed of light, a is the semi major axis of Mars orbit (in meters), e is the eccentricity.

\section{Appendix B}

\section{Calculation of Gravitational Time Dilation}

1). Calculation of Gravitational Time Dilation Relative to GPS Satellites (Worked Example)

$$
\begin{gathered}
\Delta t=3 G M /\left(c^{2} R\right) \\
G M_{E}\left(c^{2}\right)=4.436 \times 10^{-3}
\end{gathered}
$$

The total radius $R$ is given as the altitude of GPS $=20,184 \mathrm{~km}$, plus the radius of the Earth $R_{E}=6,372 \mathrm{~km}$. Thus for the GPS satellite $\mathrm{R}=26,556 \mathrm{~km}$ :

$$
\Delta t=G M_{\mathrm{E}} /\left(c^{2} R\right)=1.670 \times 10^{-10}
$$


For Earth bound obersvers:

$$
\Delta t=G M_{\mathrm{E}} /\left(c^{2} R_{\mathrm{E}}\right)=6.959 \times 10^{-10}
$$

The time difference ratio for the satellite is:

$$
6.959 \times 10^{-10}-1.670 \times 10^{-10}=5.289 \times 10^{-10}
$$

Multiplied by the number of seconds in a day 86,400

$$
\Delta_{t}=45.7 \mu \mathrm{sec} / \mathrm{day}
$$

Observed gravitational time dilation relative to GPS, $\Delta_{\mathrm{t}} \approx 45 \mu \mathrm{sec} /$ day.

where $\Delta_{t}$ is the change in time, $G$ is the gravitational constant, $M_{E}$ is the mass of the Earth, $R_{E}$ is the radius of Earth, $R$ is the total orbital Radius, $c$ the speed of light. 